Supporting Information for

\title{
Nano GeTe embedded in a three-dimensional carbon sponge for flexible Li-ion and Na-ion battery anodes
}

Tianbiao Zeng ${ }^{\mathrm{a}, \mathrm{c}}$, Dong Feng*b,a, Qimeng Peng ${ }^{\mathrm{c}}$, Qi Liu ${ }^{\mathrm{b}}$, Guocui Xic ${ }^{\mathrm{c}}$, Gang Chen*d,a

a. State Key Laboratory of Polymer Materials Engineering, Polymer Research Institute of Sichuan University, Chengdu, 610065, China

b. Faculty of Chemical Engineering, Kunming University of Science and Technology, Kunming, 650500, China

c. College of Chemistry and Chemical Engineering, Chongqing University of Technology, Chongqing 400054, China

d. School of Materials Science and Engineering, Xihua University, Chengdu, 610039, Sichuan, China

*: Corresponding authors, fdryan@kust.edu.cn, gangchen@,mail.xhu.edu.cn 
Fig. S1 XRD patterns of graphite and milled graphite.

Fig. S2 Specific surface area of (a) graphite and (b) milled graphite. 
Fig. S3 TG curves of GeTe and GeTe/G.

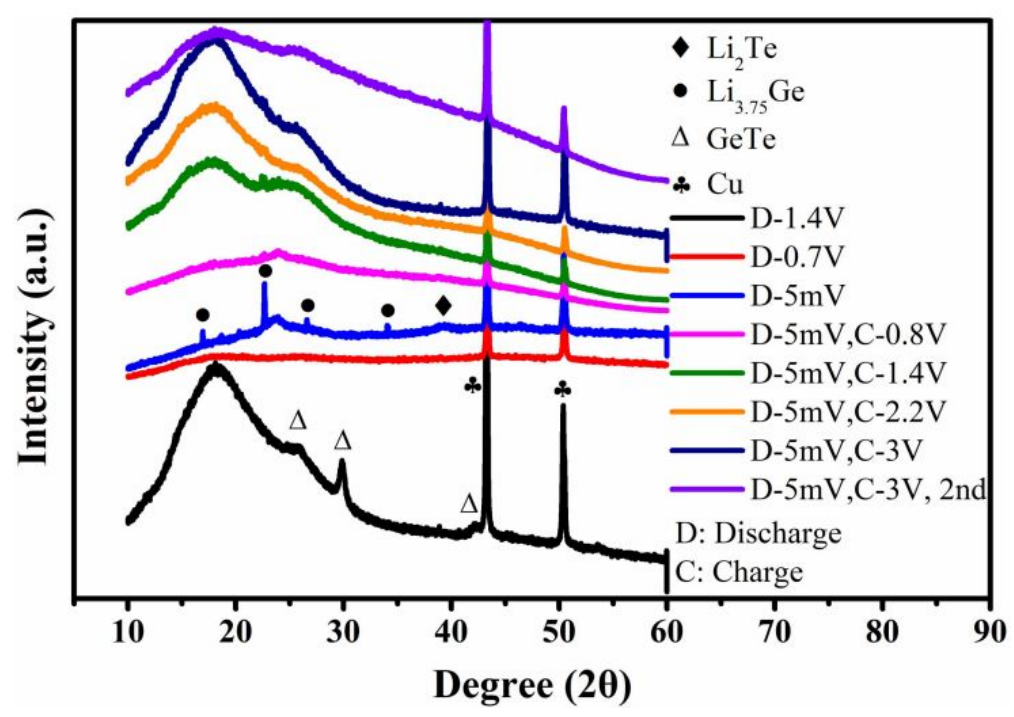

Fig. S4 EX-XRD patterns during charge/discharge cycling.

When the prime GeTe/G was discharged to $1.4 \mathrm{~V}$, broad diffraction peaks of GeTe were detected, indicating that small amount of $\mathrm{Li}^{+}$was inserted into the lattice of GeTe and GeTe crystal was swollen. When discharged to $5 \mathrm{mV}, \mathrm{Li}_{2} \mathrm{Te}$ and $\mathrm{Li}_{3.75} \mathrm{Ge}$ were detected. Then, when charged to $0.8 \mathrm{~V}$, the diffraction peaks of $\mathrm{Li}_{2} \mathrm{Te}$ and $\mathrm{Li}_{3.75} \mathrm{Ge}$ were vanished, indicating that $\mathrm{Li}$ was extracted from $\mathrm{Li}_{2} \mathrm{Te}$ and $\mathrm{Li}_{3.75} \mathrm{Ge}$. Ki-Hun Nam et al. proved that the $\mathrm{Li}_{3.75} \mathrm{Ge}$ was converted to nano Ge at 
this state, and small amount of $\mathrm{Li}$ was extracted from $\mathrm{Li}_{2} \mathrm{Te}$ to form $\mathrm{Li}_{2-x} \mathrm{Te}$ (via ex-XRD and X-Ray Absorption Fine Structure measurement). When the electrode was charged to $3 \mathrm{~V}$, no diffraction peaks can be distinguished except the $\mathrm{Cu}$ peaks, however, the $\mathrm{CV}$ curves showed a sharp and broad peak at $\sim 1.80 \mathrm{~V}$, indicating that the nano $\mathrm{Ge}$ and $\mathrm{Li}_{2-x} \mathrm{Te}$ was converted to GeTe. The GeTe content was extremely small, and no diffraction peaks can be distinguished from ex-XRD [S1].

Fig. S5 CV curves in the initial five cycles for (a) GeTe and (b) GeTe+G. 
Fig. S6 Cycle capacities and coulombic efficiency of Ge+Te, GeTe, GeTe+G and G.

Fig. S7 (a) EIS curves of Ge+Te, GeTe, GeTe+G, GeTe/G; (b) GITT profiles of Ge+Te, GeTe, $\mathrm{GeTe}+\mathrm{G}$, and GeTe/G; (c) voltage against square root of relaxation time; (d) $\mathrm{D}_{\mathrm{Li}^{+}}$of $\mathrm{Ge}+\mathrm{Te}$, GeTe, $\mathrm{GeTe}+\mathrm{G}$, and $\mathrm{GeTe} / \mathrm{G}$. 

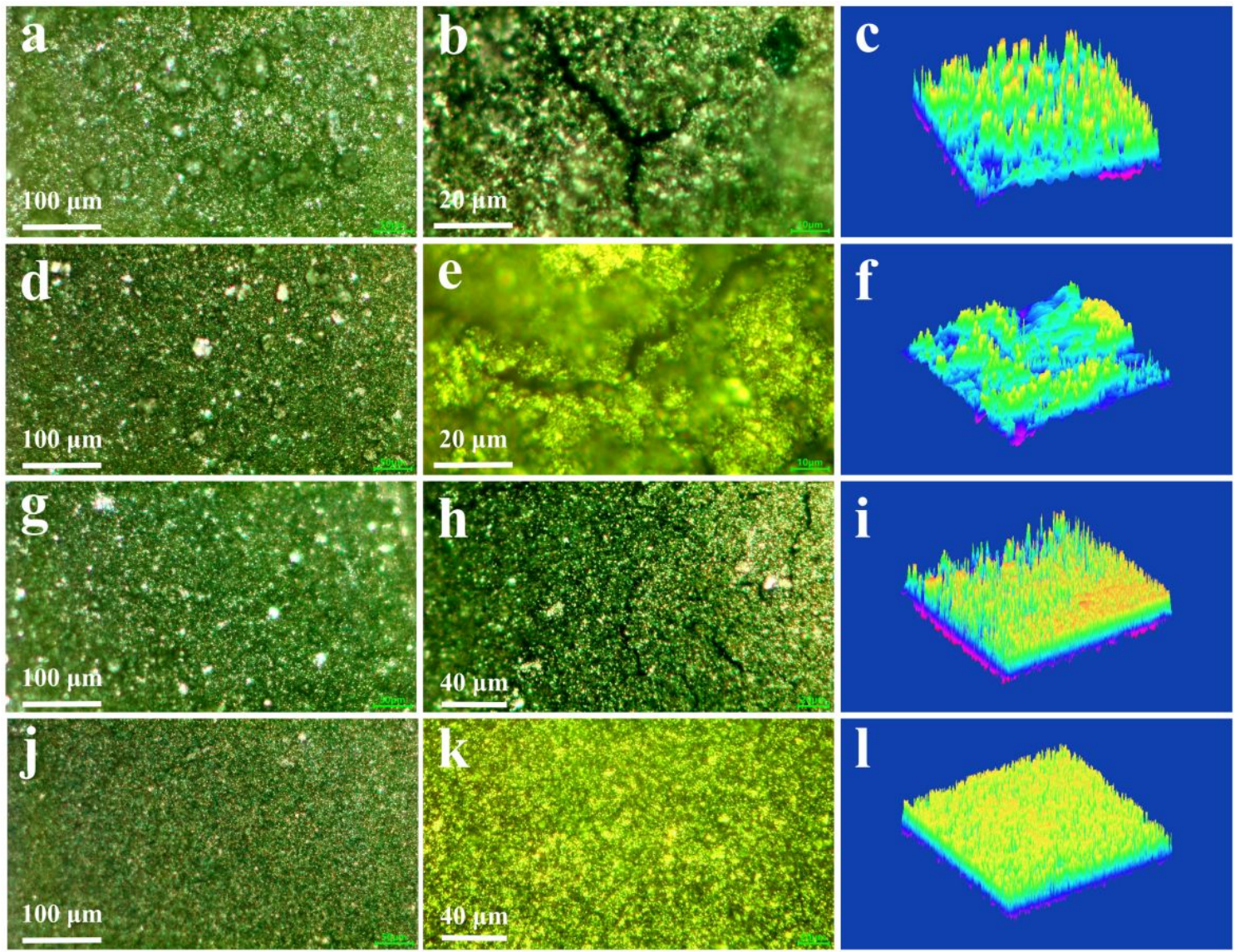

Fig. S8 Optical microscope images of electrodes: (a) primary Ge+Te, (b) cycled Ge+Te, (d) primary GeTe, (e) cycled GeTe, (g) primary $\mathrm{GeTe}+\mathrm{G}$, (h) cycled GeTe+G, (j) primary GeTe/G, (k) cycled GeTe/G; 3D height distribution histograms of cycled electrodes: (c) Ge+Te, (f) GeTe, (i) GeTe+G, (1) GeTe/G. 

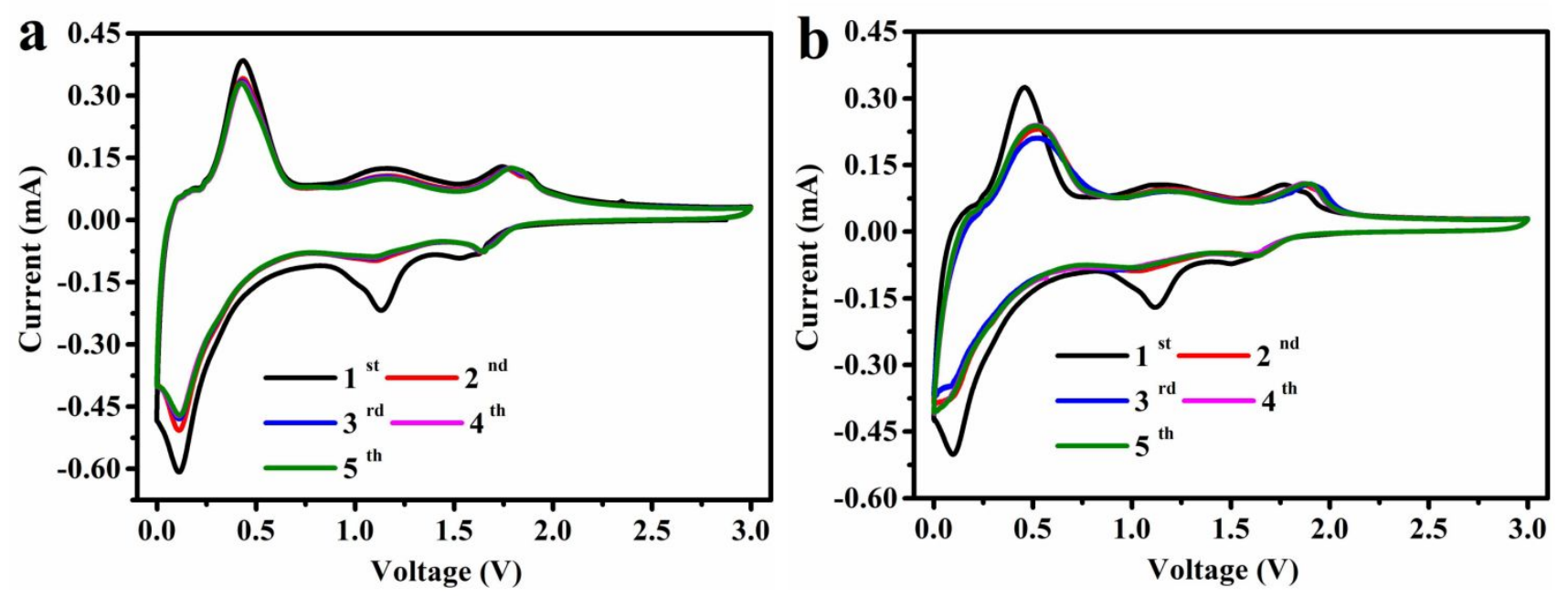

Fig. S9 CV curves in the initial five cycles for (a) FTED-1 and (b) FTED-2.

Fig. S10 Discharge/charge profiles of (a) FTED-1 and (b) FTED-2. 
Fig. S11 TG curves of cotton cellulose.

Fig. S12 Rate CV profiles of (a) GeTe/G, (b) FTED-1 and (c) FTED-2.

Fig. S13 Rate CV profiles of (a) GeTe/G and (b) FTED-3. 
Reference

[S1] F. Shojaei, H.S. Kang, Electronic structure of germanium phosphide monolayer and Li-diffusion in its bilayer, Phys. Chem. Chem. Phys. 18 (2016) 32458-32465. https://doi.org/10.1039/C6CP06090K. 